\title{
Is Lugol necessary for endoscopic resection of esophageal squamous cell neoplasia?
}

\section{다)(앙}

\section{Authors}

Maria Pia Costa-Santos ${ }^{1}$, Alexandre Oliveira Ferreira' ${ }^{1}$, Christina Mouradides ${ }^{2}$, Enrique Pérez-Cuadrado-Robles ${ }^{2}$, Ralph Yeung $^{2}$, Rodrigo Garcés-Duran ${ }^{2}$, Christophe Snauwaert ${ }^{2}$, Hélène Dano ${ }^{3}$, Hubert Piessevaux ${ }^{2}$, Pierre H. Deprez ${ }^{2}$

Institutions

1 Gastroenterology Department, Hospital Beatriz Ângelo, Loures, Portugal

2 Gastroenterology and Hepatology Department, Cliniques universitaires Saint-Luc, Université Catholique de Louvain, Brussels, Belgium

3 Pathology Department, Cliniques universitaires SaintLuc, Université Catholique de Louvain, Brussels, Belgium

submitted 16.12.2019

accepted after revision 22.5.2020

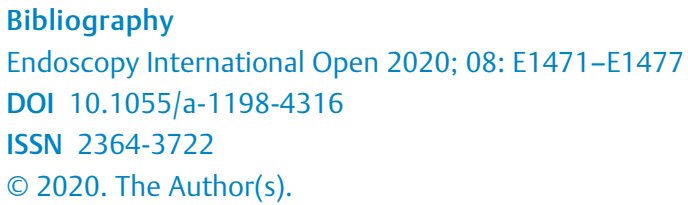

This is an open access article published by Thieme under the terms of the Creative Commons Attribution-NonDerivative-NonCommercial License, permitting copying and reproduction so long as the original work is given appropriate credit. Contents may not be used for commecial purposes, or adapted, remixed, transformed or built upon. (https://creativecommons.org/licenses/by-nc-nd/4.0/)

Corresponding author

Maria Pia Costa-Santos, Gastroenterology Department, Hospital Beatriz Ângelo, Avenida Carlos Teixeira, 3-2674-514 Loures, Portugal

Fax: +351219847 209

mariapiacostasantos@gmail.com

\section{ABSTRACT}

Background and study aims Recent evidence suggests that lugol chromoendoscopy (LCE) and narrow-band imaging (NBI) have comparable sensitivity for detection of superficial esophageal squamous cell carcinoma (SCC). However, LCE is time-consuming and associated with side effects. The aim of this study was to compare the effectiveness of NBI and LCE in defining resection margins of esophageal SCC.

Patients and methods This was a retrospective observational cohort study of patients with esophageal SCC and dysplasia who underwent en-bloc resection between 1999 and 2017 at the Cliniques universitaires Saint-Luc, Brussels. Two groups were defined: 1) inspection with NBI only; and 2) inspection with LCE (with or without NBI). The primary endpoint was complete lateral resection rate. Multivariate regression was used to adjust for potential confounders.

Results A total of 102 patients with 132 lesions were included. Lesions were inspected with LCE in $52 \%(n=68)$ and with $\mathrm{NBI}$ only in $48 \%(n=64)$. Lesions 0 -Ila were more frequent in the NBI group (37\%) and 0 -IIb (60\%) in LCE. Lesion location, size, and histology and resection technique (endoscopic submucosal dissection in 122/132 cases, 92\%) were similar between the groups. The rate of complete lateral resection for invasive carcinoma was $90 \%$ in LCE group and $94 \%$ in NBI group $(P=0.498)$ and $65 \%$ and $67 \%(P=$ $0.813)$, respectively, for dysplasia complete lateral resection. These results remained non-significant after adjusting for potential confounders.

Conclusions Mucosal inspection and delineation of tumors with lugol chromoendoscopy before endoscopic resection of esophageal squamous cell lesions was not associated with increased complete lateral resection rate when compared to NBI.

\section{Introduction}

Esophageal cancer is the eighth most common cancer and the sixth leading cause of cancer-related mortality in the world [1]. Squamous cell carcinoma (SCC) is the most frequent histologi- cal subtype accounting for $90 \%$ of cases of esophageal cancer worldwide due to its high prevalence in Eastern Asia [1-3].

Detection and characterization of early stage esophageal SCC can be challenging, since it typically appears as subtle and flat lesions on conventional white light endoscopy [1,4]. Lugol dye chromoendoscopy (LCE) has long been the gold standard 
for detection of superficial SCC and it is based on the lack of absorption of the iodine stain by abnormal squamous tissue $[3,5$, $6]$. However, lugol staining can cause heartburn and chest discomfort, has risk of pulmonary aspiration and allergic reaction, and increases the duration of the procedure $[3,6]$. Moreover, LCE is a highly sensitive but not very specific technique for detecting superficial esophageal SCC since both inflamed and dysplastic/neoplastic lesions can appear as iodine-unstained areas [4]. Therefore, new technologies have been developed to evaluate the esophageal mucosa of high-risk patients, such as narrow-band imaging (NBI, Olympus) $[7,8]$. $\mathrm{NBI}$ is a virtual chromoendoscopy technique that enhances the mucosa surface and the underlying capillary pattern simply by pressing a button [9]. Early squamous cell lesions can be identified by this technology as brownish, well demarcated lesions [3]. Disadvantages of this technique include the high cost of the device, which decreases accessibility, and its' application requires expertise [3].

Several studies compared the accuracy of NBI and LCE for diagnosing esophageal SCC $[4,10,11]$. A recent meta-analysis showed that NBI has a comparable sensitivity to that of lugol chromoendoscopy ( $88 \%$ vs. $92 \%$ ), and a superior specificity ( $88 \%$ vs. $82 \% P<0.001$ ) [12]. However, to our knowledge, there are no data on the effectiveness of NBI in delineating esophageal SCC margin before endoscopic resection.

Therefore, the aim of this study was to compare the effectiveness of NBI and LCE in defining resection margins of esophageal squamous cell cancer and dysplasia. Our hypothesis was that NBI may be equivalent to LCE in determining esophageal SCC limits prior to endoscopic resection.

\section{Patients and methods}

We conducted a retrospective observational cohort study of all patients with esophageal SCC and dysplasia who underwent enbloc resection between 1999 and 2017 at the Cliniques Universitaires Saint-Luc, Brussels, Belgium. The study protocol was approved by our ethical review board in April 2014 (2014/30AVR/ 210). The study protocol conforms to the ethical guidelines of the 1975 Declaration of Helsinki as reflected in the approval by the Comité d'Ethique Hospitalo-Facultaire Université Catholique de Louvain.

Two groups were defined before endoscopic resection: 1) inspection of esophageal lesions exclusively with NBI; and 2) inspection with lugol chromoendoscopy (with or without NBI) ( Fig.1). The decision to perform each technique was based on availability and endoscopist choice. In the first group, abnormal mucosal areas were identified and resection margins defined by NBI. When NBI was used, brown-stained areas of the mucosa were considered lesions suspected to be neoplasia (compared to "normal" mucosa, which is green independently of changes in surface or vascular texture). Delineation was mainly based on differences between green and brown mucosa, but included analysis of the intrapapillary capillary loop (IPCLs) either considered as normal (type A) vs abnormal (type B1-3), when magnification was available [7]. In the other cases, iodine solution ( $2 \%$ ) was sprayed over the entire esophageal mucosa and areas clearly not stained were suspected to be neoplasia, which is characterized by a yellow color in contrast with brown "normal" areas. The pink sign was not used for delineation since it is usually more centrally seen in the tumour. Delineation of the lesions was always performed immediately before the resection. Markings were place $2 \mathrm{~mm}$ apart from the delineation line and incision a further $2 \mathrm{~mm}$ away from markers ( Fig. 2).

Procedures were carried out using Olympus scopes (series 140 and 160 without NBI capability, 180 and 190 including HQ190 with near focus) by six experienced endoscopists. Procedures were performed under deep sedation (propofol) or anesthesia with endotracheal intubation. After delineation of lesions margins, they were resected by en-bloc endoscopic mucosal resection (EMR) or endoscopic submucosal dissection (ESD). ESD was the preferred resection technique whenever there was a suspicion of superficial invasive SCC (intramucosal or submucosal) aiming at complete lateral and deep resection (R0 resection) ( $\mathbf{F i g . ~} \mathbf{3})[13,14]$.

Patient demographic and clinical characteristics were registered including age, gender, smoking and alcohol habits, and previous treatments (EMR, ESD and/or radiotherapy). During endoscopy, lesion location (upper, middle and lower third of the esophagus), size and morphology according to Paris Classification were assessed. We also registered the endoscopist performing the procedure, the scope model and the resection technique (en-bloc EMR or ESD). Histology was assessed and registered according to the following four categories of the World Health Organization (WHO) Classification of tumors of the digestive system: invasive SCC (tumor invading the lamina propria), high-grade intraepithelial neoplasia/dysplasia (HGD) (includes all non-invasive intraepithelial carcinomas formerly called carcinoma in situ), low-grade intraepithelial neoplasia/ dysplasia (LGD), and negative for neoplasia/dysplasia (no atypia) [15]. Lateral margins were evaluated in all lesions. In case of invasive SCC, depth of invasion (T1a [intramucosal] and T1b [submucosal]), tumor differentiation, and presence of lymphovascular invasion were also recorded $[15,16]$. Need for additional treatment (EMR, ESD, surgery, radiotherapy or chemotherapy) and recurrence rate were also evaluated.

Complete lateral resection rate was defined as cancer/dysplasia free margin where applicable. Complete resection (R0) was defined as resection with lateral and vertical margin free of cancer and dysplasia. Curative resection was evaluated in cases of invasive SCC and defined as lesions that did not need additional treatment. Recurrence was considered local when a new lesion was diagnosed on a scar of a previous resection and metachronous if it occurred in another location.

The primary endpoint was complete lateral resection rate cancer and dysplasia-free margin. Secondary endpoints were $\mathrm{R} 0$ resection and recurrence rates.

\section{Statistical analysis}

All continuous variables were described as mean and standard deviation or median and range while categorical variables were expressed as frequency and proportions. In case of missing data, the denominator was adjusted to calculate the correct 


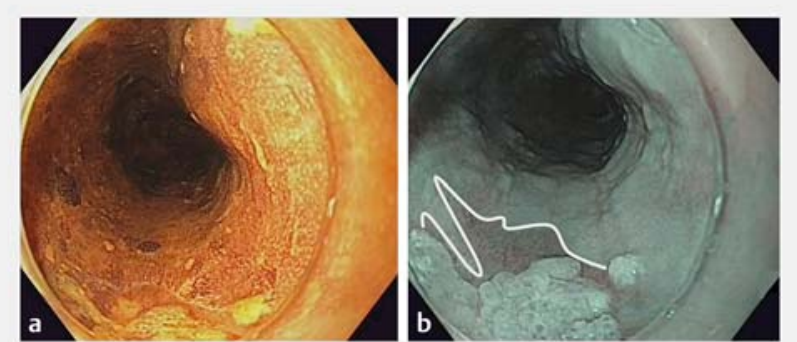

- Fig.1 Esophageal lesion with lugol chromoendoscopy on the left and NBI on the right. The delineated area identified with NBI is highgrade dysplasia (brown and flat, below the nodular whitish lesion) and could have been missed with lugol chromoendoscopy showing normally stained mucosa at the dysplastic site.

proportions. Mean differences of continuous variables with a normal distribution were analysed using an independent Student $\mathrm{t}$-test. The other continuous variables were compared using the Wilcoxon Mann-Whitney test. To explore univariate associations in the distribution of categorical data, the $\mathrm{X}^{2}$ test or Fisher's exact test was used as appropriate. For the primary end-point - complete lateral resection - a multiple logistic regression was used to adjust for potential confounders. $P<0.05$ was considered statistically significant. Statistical analysis was performed using the software Statistical Package for Social Sciences (SPSS) (version 23.0).

\section{Results}

During the study period, 102 patients were included, 65.7\% male, with a mean age of $64.9 \pm 9.4$ years. $>$ Table 1 shows patient demographic and clinical characteristics.

In total, 132 lesions were resected, with a median lesions per patient of $1(1-6)$ ( $\triangleright$ Table 2$)$. In $52 \%(n=68)$, resection margins were defined by LCE (with or without NBI) and in $48 \%(n=$ 64) by NBI exclusively. The endoscopy was performed by the same endoscopist in $71 \%$ of cases. A H190 or HQ190 scope was used in more than half of patients. About two-thirds of

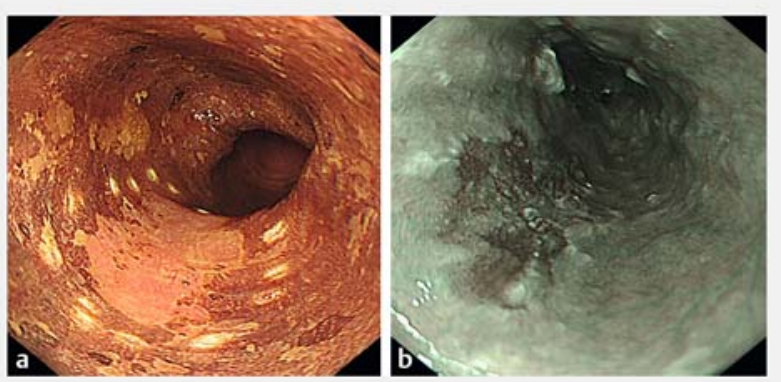

- Fig. 2 Esophageal lesion with lugol chromoendoscopy on the left (iodine-unstained area) and NBI on the right (brown). Markings were place $2 \mathrm{~mm}$ apart from de delineation line (on the left).

the lesions were in the middle third of the esophagus, with a median size of $30 \mathrm{~mm}(5-100)$. Lesions were classified as 0 -Ila in $29 \%$ of cases and as $0-1 \mathrm{lb}$ in $42 \%$. In total $92 \%(n=122)$ of the lesions were resected by ESD and pathological analysis revealed invasive SCC in $77 \%(n=101)$. In invasive SCC lesions, $64 \%$ were T1a and $36 \% \mathrm{~T} 1 \mathrm{~b}, 42 \%$ (39/93) and $11 \%$ (10/93) were well and poorly differentiated, respectively, and lymphovascular invasion was present in $25 \%(15 / 61)$.

The rate of complete lateral resection for invasive carcinoma was $92 \%(93 / 101)$ and $66 \%(86 / 131)$ for dysplasia. Complete lateral and deep resection ( $\mathrm{R} 0$ resection) was achieved in $49 \%$ (64/130). Incomplete R0 resection (66/130) was due to lateral invasion in $53 \%(n=35)$, deep invasion in $32 \%(n=21)$ and both lateral and deep in $15 \%(n=10)$. The curative resection rate was $80.5 \%$.

Of the 102 patients, 21 underwent additional treatment: one with EMR, one with ESD, eight underwent surgery, one radiotherapy and, 10 chemoradiotherapy.

The median time of follow-up was 13 months $(0-154)$. Local recurrence rate was $3 \%(n=4)$ after a median time of follow-up of 16 months (1-17).
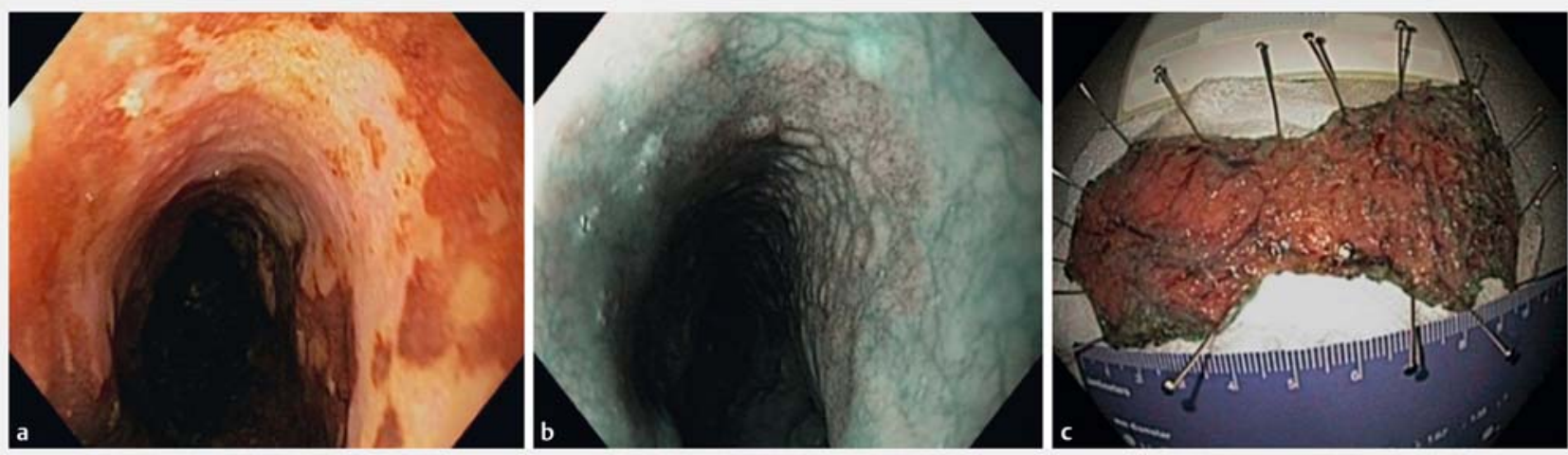

- Fig. 3 Esophageal lesion with lugol chromoendoscopy on the left (iodine-unstained area) and NBI in the middle (brown). Stretched resected specimen on the right. 
- Table 1 Patient demographic and clinical characteristics $(n=102)$.

\begin{tabular}{|c|c|c|c|c|}
\hline & Total $(n=102)$ & LCE $(n=52)$ & NBI $(n=50)$ & $P$ value \\
\hline Age - mean $\pm S D$, years & $64.9 \pm 9.4$ & $63.4 \pm 9.7$ & $66.4 \pm 8.7$ & 0.110 \\
\hline Male gender - n (\%) & $67(66 \%)$ & $39(75 \%)$ & $28(56 \%)$ & 0.043 \\
\hline \multicolumn{5}{|l|}{ Smoking habits - n (\%) ${ }^{1}$} \\
\hline Never smoker & $20(21 \%)$ & $7(14 \%)$ & $13(28 \%)$ & \multirow[t]{2}{*}{0.107} \\
\hline Ever Smoker & $76(79 \%)$ & $42(86 \%)$ & $34(72 \%)$ & \\
\hline \multicolumn{5}{|l|}{ Drinking habits - n $(\%)^{2}$} \\
\hline Yes & $69(71 \%)$ & $43(86 \%)$ & $26(55 \%)$ & \multirow[t]{2}{*}{0.001} \\
\hline No & $28(29 \%)$ & $7(14 \%)$ & $21(45 \%)$ & \\
\hline \multicolumn{5}{|c|}{ Previous treatment - $\mathrm{n}(\%)$} \\
\hline EMR/ESD & $7(7 \%)$ & $5(10 \%)$ & $2(4 \%)$ & \\
\hline Radiotherapy & $21(21 \%)$ & $14(27 \%)$ & $7(14 \%)$ & $\mathrm{N} / \mathrm{A}$ \\
\hline EMR and radiotherapy & $3(3 \%)$ & $2(4 \%)$ & $1(2 \%)$ & \\
\hline \multicolumn{5}{|l|}{ Number of lesions - $n(\%)$} \\
\hline 1 & $87(85 \%)$ & $44(85 \%)$ & $43(86 \%)$ & \\
\hline $2-3$ & $13(13 \%)$ & $7(13 \%)$ & $6(12 \%)$ & $\mathrm{N} / \mathrm{A}$ \\
\hline$\geq 4$ & $2(2 \%)$ & $1(2 \%)$ & $1(2 \%)$ & \\
\hline \multicolumn{5}{|c|}{$\begin{array}{l}\text { SD, standard deviation; LCE, Lugol chromoendoscopy; NBI, narrow-band imaging; EMR, endoscopic mucosal resection; ESD, endoscopic submucosal dissection; N/A } \\
\text { not applicable. } \\
P \text { value comparing LCE group versus NBI group. } \\
{ }^{1} \text { Missing data from } 6 \text { patients. } \\
{ }^{2} \text { Missing data from } 5 \text { patients. }\end{array}$} \\
\hline
\end{tabular}

\section{Lugol chromoendoscopy versus NBI}

There were more male patients in the LCE group than in the NBI group ( $75 \%$ vs. $56 \%, P=0.043$ ). There were no differences in patient's age between groups.

- Table 2 shows endoscopy and lesions characteristics in both groups. In the NBI group lesions 0-Ila were more frequent (37\%) whereas in the LCE group were 0 -IIb (60\%). Lesions location and size and resection technique were similar between groups. The scope model was $\mathrm{H} 180 / \mathrm{H} 190 / \mathrm{HQ} 190$ in all patients in the NBI group ( $100 \%$ vs. $91 \%, P=0.028)$. Median follow-up time was longer in the LCE group (18. vs 8 months, 0.006).

The rate of complete lateral resection for invasive carcinoma was $90 \%$ in the LCE group and $94 \%$ in NBI group $(P=0.715)$ and $65 \%$ and $67 \%(P=0.813)$, respectively, for dysplasia complete lateral resection ( $\triangleright$ Table 3 ). On multiple logistic regression, the results were similar after adjusting for previous local treatment, scope model, lesion size, morphology and histology and resection technique (Odds ratio [OR] 0.544, $95 \%$ Confidence interval $[95 \% \mathrm{Cl}] 0.160-1.847, P=0.329$ for cancer complete lateral resection; OR $0.590,95 \% \mathrm{Cl} 0.219-1.592, P=0.298$ for dysplasia complete lateral resection).

Complete lateral and deep resection for cancer and dysplasia was $45 \%$ and $54 \%$ in LCE and NBI group, respectively $(P=0.295)$
There were four cases of local recurrence: three (4\%) in the LCE group and one $(2 \%)$ in the NBI group $(P=0.627)$. Additional treatment was necessary in $15 \%$ of patients in LCE group (one EMR, one ESD, four surgery and four chemoradiotherapy) and in $17 \%$ in the $\mathrm{NBI}$ group (four surgery, one radiotherapy and six chemoradiotherapy) $(\mathrm{P}=0.697)$.

\section{Predictive factors of complete lateral resection}

An exploratory analysis was performed to identify predictive factors for complete lateral resection for invasive carcinoma and dysplasia, namely previous local treatment, scope model, lesion location, size, morphology and histology and resection technique. The scope model (H180, H190 and HQ190 vs. 140 and 160 series) was the only factor associated with a higher cancer and dysplasia-free lateral margin (68\% vs $17 \%, P=$ 0.017).

\section{Discussion}

Herein, for the first time we compared the effectiveness of NBI and LCE in defining resection margins for esophageal SCC and dysplasia. We found that mucosal inspection with LCE before endoscopic resection of esophageal squamous cell lesions was not associated with increased complete lateral resection rate when compared to NBI alone. The rate of complete lateral and deep resection was also similar between groups. Furthermore, 
- Table 2 Endoscopy and lesion characteristics $(n=132)$.

\begin{tabular}{|c|c|c|c|c|}
\hline & Total $(n=132)$ & LCE $(n=68)$ & NBI $(n=64)$ & $P$ value \\
\hline \multicolumn{5}{|l|}{ Endoscopist - n (\%) } \\
\hline P.D. & $94(71 \%)$ & $47(69 \%)$ & $47(73 \%)$ & \multirow[t]{6}{*}{$\mathrm{N} / \mathrm{A}$} \\
\hline H.P. & $15(11 \%)$ & $9(13 \%)$ & $6(9 \%)$ & \\
\hline R.Y. & $12(9 \%)$ & $11(16 \%)$ & $1(2 \%)$ & \\
\hline C.S. & $5(4 \%)$ & $0(\%)$ & $5(8 \%)$ & \\
\hline R.D. & $5(4 \%)$ & $0(0 \%)$ & $5(8 \%)$ & \\
\hline T.A. & $1(1 \%)$ & $1(2 \%)$ & $0(0 \%)$ & \\
\hline \multicolumn{5}{|l|}{ Scope model - n (\%) ${ }^{1}$} \\
\hline $140 / 160$ & $6(5 \%)$ & $6(9 \%)$ & $0(0 \%)$ & \\
\hline $\mathrm{H} 180$ & $51(39 \%)$ & $39(59 \%)$ & $12(19 \%)$ & $<0.001$ \\
\hline H190/HQ190 & $73(56 \%)$ & $21(32 \%)$ & $52(81 \%)$ & \\
\hline \multicolumn{5}{|l|}{ Lesion location - n (\%) } \\
\hline Upper third & $25(18.9 \%)$ & $12(18 \%)$ & $13(20 \%)$ & \\
\hline Middle third & $80(60.6 \%)$ & $43(63 \%)$ & $37(58 \%)$ & 0.816 \\
\hline Lower third & $27(20.5 \%)$ & $13(19 \%)$ & $14(22 \%)$ & \\
\hline Lesion size - median \pm range, $\mathrm{mm}$ & $30(5-100)$ & $22(5-90)$ & $30(5-100)$ & 0.123 \\
\hline \multicolumn{5}{|l|}{ Lesion morphology - n (\%) } \\
\hline 0 -Is & $6(5 \%)$ & $2(3 \%)$ & $4(7 \%)$ & \multirow[t]{7}{*}{$\mathrm{N} / \mathrm{A}$} \\
\hline 0 -Ila & $36(29 \%)$ & $13(21 \%)$ & $23(37 \%)$ & \\
\hline $0-I I b$ & $53(42 \%)$ & $38(60 \%)$ & $15(24 \%)$ & \\
\hline 0 -IIc & $4(3 \%)$ & $2(3 \%)$ & $2(3 \%)$ & \\
\hline $0-11 a+11 b$ & $6(5 \%)$ & $4(6 \%)$ & $2(3 \%)$ & \\
\hline $0-11 a+\| c$ & $16(13 \%)$ & $3(5 \%)$ & $13(21 \%)$ & \\
\hline $0-\|I b+\| c$ & $4(3 \%)$ & $1(2 \%)$ & $3(5 \%)$ & \\
\hline \multicolumn{5}{|l|}{ Resection technique - n (\%) } \\
\hline ESD & $122(92 \%)$ & $64(94 \%)$ & $58(91 \%)$ & \multirow[t]{2}{*}{0.522} \\
\hline EMR & $10(8 \%)$ & $4(6 \%)$ & $6(9 \%)$ & \\
\hline \multicolumn{5}{|l|}{ Lesion histology - n (\%) } \\
\hline Invasive SCC & $101(76.5 \%)$ & $51(75 \%)$ & $50(78 \%)$ & \multirow[t]{3}{*}{ N/A } \\
\hline High-grade dysplasia & $25(18.9 \%)$ & $14(21 \%)$ & $11(17 \%)$ & \\
\hline Low-grade dysplasia & $6(4.5 \%)$ & $3(4 \%)$ & $3(5 \%)$ & \\
\hline Follow-up - median \pm range, months & $13(0-154)$ & $18(0-154)$ & $8(0-40)$ & 0.006 \\
\hline \multicolumn{5}{|c|}{$\begin{array}{l}\text { LCE, Lugol chromoendoscopy; NBI, narrow-band imagi } \\
\text { noma; N/A, not applicable. } \\
\text { P value comparing LCE group versus NBI group. } \\
{ }^{1} \text { Missing data from two lesions. } \\
{ }^{2} \text { Paris Classification, missing data from seven lesions. }\end{array}$} \\
\hline
\end{tabular}

there were no differences in recurrence rate and need of additional therapies.

Esophageal cancers carry a high mortality mainly due to diagnosis in advanced-stage, with a five-year survival rate of less than $10 \%$ [17]. Patients with superficial esophageal SCC, confined to the mucosa or submucosa, have a significantly better prognosis [17], with a 5-year survival rate exceeding $85 \%$ for PT1N0 lesions [18]. However, diagnosis of early stage esopha- 


\begin{tabular}{|c|c|c|c|}
\hline & LCE $(N=68)$ & NBI $(N=64)$ & $P$ value \\
\hline Cancer-free lateral margin - $\mathbf{n}(\%)^{1}$ & $46(90 \%)$ & $47(94 \%)$ & 0.715 \\
\hline Dysplasia free lateral margin - $n(\%)^{2}$ & $44(65 \%)$ & $42(67 \%)$ & 0.813 \\
\hline $\mathrm{RO}$ resection $-\mathrm{n}(\%)^{3}$ & $30(45 \%)$ & $34(54 \%)$ & 0.295 \\
\hline Local recurrence - n (\%) & $3(4 \%)$ & $1(2 \%)$ & 0.627 \\
\hline Metachronous lesions - n (\%) & $11(16 \%)$ & $6(9 \%)$ & 0.244 \\
\hline \multicolumn{4}{|c|}{$\begin{array}{l}\text { LCE, Lugol chromoendoscopy; NBI, narrow-band imaging. } \\
1 \text { Included only invasive squamous cell carcinoma }(n=101) \\
{ }^{2} \text { Missing data from one lesion. } \\
{ }^{3} \text { Missing data from two lesions. }\end{array}$} \\
\hline
\end{tabular}

geal SCC and their precursor lesions can be difficult because of their flat and isochromatic appearance on conventional whitelight imaging endoscopy $[1,4]$. Lugol staining has long been the gold standard for detection of these lesions [5, 6]. Although it is a low-cost technique, it may have side effects and lead to complications [3,6]. Therefore, new diagnostic strategies based on virtual chromoendoscopy, such as NBI, have been used for detection of superficial esophageal squamous cells carcinoma and dysplasia $[7,8]$.

Several studies have compared the accuracy of NBI and LCE for diagnosing esophageal SCC $[4,10,11]$. Only one prospective randomized controlled trial (RCT) have been published on this subject [4]. In this study, Goda et al compared the diagnostic accuracy of NBI magnifying endoscopy (NBI-ME) and lugol chromoendoscopy with pink-color sign assessment (LCE-PS) for superficial esophageal SCC in 294 patients, 147 in each arm. In per-patient analysis, there were no differences between NBI-ME and LCE-PS in sensitivity ( $82 \%$ vs $81 \%$ ), specificity ( $95 \%$ vs $94 \%$ ), positive predictive value ( 88 vs $85 \%$ ), negative predictive value ( $92 \%$ vs $93 \%$ ), and overall accuracy (91 vs $91 \%$ ) for diagnosing superficial esophageal SCC. Median examination time with LCE-PS was 1.5 times longer than NBI-ME $(P<0.001)$. More recently, a meta-analysis compared the diagnostic accuracy of NBI and LCE in identifying esophageal HGD and/or SCC [12]. Twelve studies were included, 11 cross-sectional and one RCT, and 1911 patients were analyzed. The authors showed that NBI has a comparable sensitivity to that of LCE in both per-patient and per-lesion analysis ( $88 \%$ vs $92 \%$ and $94 \%$ vs. $98 \%$, respectively), and a superior specificity ( $88 \%$ vs. $82 \%, P<$ 0.001 and $65 \%$ vs $37 \%, P<0.001$, respectively). Therefore, NBI presented the same rate of detection of esophageal HGD and SCC when compared to LCE, but was superior in differentiating HGD and SCC from other esophageal mucosa alterations. However, to our knowledge, there are no data comparing the effectiveness of NBI and LCE in delineating the esophageal SCC margin before endoscopic resection. Previous studies on superficial squamous neoplasms in the orohypopharynx showed that $\mathrm{NBI}$ is a useful tool in defining surgical resection margins of oral and oropharyngeal cancers, since it decreases the rate of positive superficial margins [19] and can also decrease recurrence and improve survival as compared to traditional methods (visual examination alone and white-light nasoendoscopy) [20].

In the current study, the complete lateral resection rate was $66 \%$ for dysplasia and $92 \%$ for invasive carcinoma, comparable to that described in two single-center and one multicenter study on endoscopic resection of early esophageal SCC (lateral margins positive for carcinoma in $8.3 \%$ to $9.5 \%$ of lesions) [2123]. It is interesting to note that local recurrence rate was inferior to complete lateral resection rate for dysplasia which may be due to pathological examination limitations due to cautery artifacts or due to cautery effect on non-resected adjacent mucosa. In the subgroup analysis in our study, the rate of complete lateral resection was similar between NBI and LCE for esophageal SCC (94\% vs $90 \%$ ) and dysplasia (67\% vs $65 \%$ ). The results were similar after adjusting for potential confounders. There were also no differences in the complete lateral and deep resection rate, relapse rate and need of additional therapies. The lower rates than expected for complete lateral resection for dysplasia might be due the fact that in the beginning of our experience we tried to avoid circumferential resections and to leave a small longitudinal band of mucosa. Other explanations may be due to the multifocal disease observed in some patients, rendering both LCE (with multiple unstained areas) and $\mathrm{NBI}$ (multiple brown areas) more difficult to assess, and the fact that $41 \%$ and $20 \%$ in LCE and NBI groups, respectively had undergone previous treatments with scarring and post-radiotherapy mucosal changes. We therefore suggest in multifocal esophageal disease to start delineation with NBI and magnification (and marking), and to use LCE only if multiple brown areas are confounding the global picture. Once LCE is used, the vascular features including IPCLs are lost. We would also like to emphasize that $\mathrm{NBI}+$ Nearfocus was able in certain cases to give more information on adjacent dysplastic mucosa (brown area) with normal LCE staining.

Our study has several limitations. First, it is a retrospective study including patients over an 18 years period. As such, different scope models were used during resection, including series 140 and 160 in LCE group which can reduce the rate of complete lateral resection in this group. In fact, we performed an exploratory analysis to detect predictive factors of complete lateral resection and more recent scope models (H180/H190/ 
HQ190) were associated with a higher cancer and dysplasiafree lateral margin. Moreover, the endoscopists were the same over the study period and therefore their skills in evaluating and resecting lesions improved over time. Second, lesion morphology was different between groups; for example, lesions 0-Ilb were more frequent in the LCE group and it might be argued that it is more challenging in to assess their lateral margins. Finally, the follow-up time is limited and was significantly longer in the LCE group, since NBI was introduced more recently; this can also influence the recurrence rate and need of additional therapies in the second group.

\section{Conclusion}

In conclusion, this is the first study comparing NBI and LCE for resection of esophageal squamous cells lesions and a considerable sample size was included. Mucosal inspection with LCE before endoscopic resection of esophageal squamous cells lesions was not associated with increased complete lateral resection rate when compared to $\mathrm{NBI}$ alone. Considering the increased availably of NBI, absence of side effects, possible shorter duration of the procedure as compared to LCE, and similar accuracy in detecting and probably delineating resection margins of SCC and precursor lesions, this technique may become the standard of care for this disease. Further confirmation of our findings in prospective randomized trials is required.

\section{Competing interests}

The authors declare that they have no conflict of interest.

\section{References}

[1] Barret M, Prat F. Diagnosis and treatment of superficial esophageal cancer. Ann Gastroenterol 2018; 31: 256-265

[2] Lordick F, Mariette C, Haustermans K et al. Oesophageal cancer: ESMO Clinical Practice Guidelines for diagnosis, treatment and followup. Ann Oncol 2016; 27: v50-v57

[3] Lopes AB, Fagundes RB. Esophageal squamous cell carcinoma - precursor lesions and early diagnosis. World J Gastrointest Endosc 2012; 4: 9-16

[4] Goda K, Dobashi A, Yoshimura N et al. Narrow-band imaging magnifying endoscopy versus lugol chromoendoscopy with pink-color sign assessment in the diagnosis of superficial esophageal squamous neoplasms: a randomised noninferiority trial. Gastroenterol Res $\mathrm{Pr}$ 2015; 2015: 639462

[5] Inoue H, Rey JF, Lightdale C. Lugol chromoendoscopy for esophageal squamous cell cancer. Endoscopy 2001; 33: 75-79

[6] Dubuc J, Winnock M, Barrioz T et al. Endoscopic screening for esophageal squamous-cell carcinoma in high-risk patients: a prospective study conducted in 62 french endoscopy centers. Endoscopy 2006; 38: 690-695

[7] Yoshida T, Inoue H, Usui S et al. Narrow-band imaging system with magnifying endoscopy for superficial esophageal lesions. Gastrointest Endosc 2004; 59: 288-295

[8] Takenaka R, Kawahara Y, Okada $\mathrm{H}$ et al. Narrow-band imaging provides reliable screening for esophageal malignancy in patients with head and neck cancers. Am J Gastroenterol 2009; 104: 2942-2948

[9] Machida H, Sano Y, Hamamoto Y et al. Narrow-band imaging in the diagnosis of colorectal mucosal lesions: a pilot study. Endoscopy 2004; 36: 1094-1098

[10] Nagami Y, Tominaga K, Machida $H$ et al. Usefulness of non-magnifying narrow-band imaging in screening of early esophageal squamous cell carcinoma: a prospective comparative study using propensity score matching. Am J Gastroenterol 2014; 109: 845-854

[11] Ide E, Maluf-filho F, Chaves DM et al. Narrow-band imaging without magnification for detecting early esophageal squamous cell carcinoma. World J Gastroenterol 2011; 17: 4408-4413

[12] Morita FHA, Bernardo WM, Ide E et al. Narrow band imaging versus lugol chromoendoscopy to diagnose squamous cell carcinoma of the esophagus: a systematic review and meta-analysis. BMC Cancer 2017; 17: 54

[13] Deprez PH, Bergman JJ, Meisner S et al. Current practice with endoscopic submucosal dissection in Europe: position statement from a panel of experts. Endoscopy 2010; 42: 853-858

[14] Pimentel-Nunes P, Dinis-Ribeiro M, Ponchon T et al. Endoscopic submucosal dissection: European Society of Gastrointestinal Endoscopy (ESGE) Guideline. Endoscopy 2015; 47: 829-854

[15] Bosman F, Carneiro F, Hruban R et al. WHO classification of tumours of the digestive system. 4th ed. Lyon: IARC; 2010

[16] Edge S, Byrd D, Compton C. AJCC cancer staging manual. 7th ed. New York: Springer; 2010

[17] Ando N. Esophageal Squamous Cell Carcinoma - Diagnosis and Treatment. Japan: Springer; 2015

[18] Tachibana M, Kinugasa S, Dhar DK et al. Prognostic factors in T1 and T2 squamous cell carcinoma of the thoracic esophagus. Arch Surg 1999; 134: 50-54

[19] Tirelli G, Piovesana M, Gatto A et al. Is NBI-guided resection a breakthrough for achieving adequate resection margins in oral and oropharyngeal squamous cell carcinoma? Ann Otol Rhinol Laryngol 2016; 125: 596-601

[20] Farah C. Narrow band imaging-guided resection of oral cavity cancer decreases local recurrence and increases survival. Oral Dis 2018; 24 : 89-97

[21] Lorenzo D, Barret M, Leblanc S et al. Outcomes of endoscopic submucosal dissection for early oesophageal squamous cell neoplasia at a Western centre. United Eur Gastroenterol J 2019; 7: 1084-1092

[22] Probst A, Aust D, Märkl B et al. Early esophageal cancer in Europe: Endoscopic treatment by endoscopic submucosal dissection. Endoscopy 2015; 47: 113-122

[23] Berger A, Rahmi G, Perrod G et al. Long-term follow-up after endoscopic resection for superficial esophageal squamous cell carcinoma: a multicenter Western study. Endoscopy 2018; 51: 298-306 